\title{
Lack of Pharmacokinetic Interaction between Oxcarbazepine and Lamotrigine
}

\author{
Jochen GW Theis*,', Jagdev Sidhu², Joanne Palmer², Sarah Job², Jonathan Bullman² and John Ascher ${ }^{3}$ \\ 'Clinical Pharmacology Unit, University of Cambridge, Cambridge, UK; ${ }^{2}$ Department of Clinical Pharmacology and Discovery Medicine, \\ GlaxoSmithKline, Harlow, UK; ${ }^{3}$ Department of Clinical Pharmacology and Discovery Medicine, GlaxoSmithKline, Research Triangle Park, NC, USA
}

\begin{abstract}
Epilepsy and bipolar disorder are commonly treated by combination drug therapy, such as lamotrigine and oxcarbazepine. To ensure the safety of this combination, information on pharmacokinetics and tolerability must be available. The objective of study was to evaluate the pharmacokinetics and tolerability of coadministered lamotrigine and oxcarbazepine in healthy subjects. This randomized, single-blind, parallel-group study comprised three cohorts: lamotrigine (200 mg daily) plus oxcarbazepine (600 mg twice daily), lamotrigine (200 mg daily) plus placebo, and oxcarbazepine (600 mg twice daily) plus placebo. Serial blood samples were collected at steady state to determine serum concentrations of lamotrigine and plasma concentrations of oxcarbazepine and its active metabolite 10-monohydroxy metabolite (MHD). Pharmacokinetic parameters were determined by noncompartmental methods. Tolerability was monitored through adverse event reports, clinical laboratory results, vital signs, and electrocardiograms. A total of 47 male volunteers received study drugs. At steady state, lamotrigine $A \cup C_{(0-24)}$ and $C_{\max }$ were not significantly affected by oxcarbazepine cotherapy, nor were $M H D A \cup C_{(0-12)}$ and $C_{\text {max }}$ significantly affected by lamotrigine cotherapy. The most common adverse events, headache, dizziness, nausea, and somnolence, occurred more frequently during lamotrigine and oxcarbazepine combination therapy than during the monotherapy. No significant changes in clinical laboratory parameters, vital signs, or electrocardiograms were reported. In conclusion, the combination of lamotrigine and oxcarbazepine does not require dose adjustments based on pharmacokinetic data. However, it is important to recognize that the combination therapy was associated with more frequent adverse events.
\end{abstract}

Neuropsychopharmacology (2005) 30, 2269-2274. doi: I 0.1038/s..npp. I 30083I; published online 27 July 2005

Keywords: lamotrigine; oxcarbazepine; cotherapy; pharmacokinetics; bipolar disorder; epilepsy

\section{INTRODUCTION}

Lamotrigine (3,5-diamino-6-(2,3,-dichlorophenyl)-1,2,4triazine) and oxcarbazepine (10,11-dihydro-10-oxo-5H-dibenz[ $b$,f]azepine-5-carboxamide) are effective antiepileptic agents (Lamictal ${ }^{\circledR}, 2004$; Trileptal $\left.{ }^{\circledR}, 2004\right)$. Both drugs are also used to treat bipolar disorder, although only lamotrigine is licensed to delay the time to occurrence of mood episodes (ie depression, mania, hypomania, and mixed episodes) (Lamictal ${ }^{\mathbb{R}}$, 2004). Although monotherapy is generally preferred for these complex diseases, patients often require polytherapy if adequate control of symptoms is not achieved (Deckers, 2002).

\footnotetext{
*Correspondence: Dr JGW Theis, Clinical Pharmacology Unit, University of Cambridge, Addenbrookes Centre for Clinical Investigation, Addenbrookes Hospital, Hills Road, Cambridge CB2 2GG, UK, Tel: + 447830 073904, Fax: + 447830610802

E-mail: Jochen_Theis@hotmail.com

Received 31 January 2005; revised 29 April 2005; accepted 10 June 2005

Online publication: 20 June 2005 at http://www.acnp.org/citations/ Npp06200505007//default.pdf
}

Oxcarbazepine is the 10-keto analogue of carbamazepine. It has similar efficacy, better tolerability, and a distinct pharmacokinetic profile compared with carbamazepine (Glauser, 2001; Shorvon, 2000). Physicians may, therefore, combine lamotrigine with oxcarbazepine to treat patients with epilepsy when insufficient seizure control is achieved with monotherapy. Additionally, this combination may be used to treat bipolar disorder as lamotrigine has been shown to prevent mood episodes with particularly robust efficacy for the prevention of depression, while oxcarbazepine reportedly has antimanic properties (Lamictal ${ }^{\circledR}, 2004$; Hirschfeld and Kasper, 2004).

During polytherapy for epilepsy, carbamazepine, a wellknown enzyme-inducer, increases the glucuronidation of lamotrigine necessitating higher lamotrigine doses to achieve adequate therapeutic plasma concentrations (Lamictal ${ }^{\mathbb{R}}$, 2004; Goa et al, 1993; Anderson et al, 2002). Conversely, oxcarbazepine is generally regarded as a weak enzyme-inducer (Tecoma, 1999); and its effects on lamotrigine pharmacokinetics are largely unknown. Two reports indicate that induction of glucuronidation pathways by oxcarbazepine may affect the metabolism of lamotrigine (May et al, 1999; Kramer et al, 2003) and another describes 
higher MHD concentrations in the presence of lamotrigine compared to oxcarbazepine monotherapy (Guenault et al, 2003). Drug interactions may also be theoretically possible due to the potential inhibition of glucuronidation, which is the first-step of the metabolism and excretion pathway of the active metabolite of oxcarbazepine 10-monohydroxy (MHD) (May et al, 2003) as well as the most important metabolic transformation inactivating lamotrigine (Cohen et al, 1987; Lloyd et al, 1994).

To investigate the potential for drug interactions between lamotrigine and oxcarbazepine and provide necessary prescribing information for physicians, we evaluated the pharmacokinetics and tolerability of this combination therapy in a bidirectional drug interaction study in healthy volunteers.

\section{METHODS}

This was a single-center, partially blinded, randomized, placebo-controlled, parallel-group study (GSK Protocol SCA10910) to investigate the potential interaction between lamotrigine and oxcarbazepine in volunteers. The study protocol was approved by an Independent Ethics Committee and the study was conducted in accordance with Good Clinical Practice.

Study participants were healthy men, 18-55 years old, who were capable of giving informed consent. At screening, participants were negative for alcohol and drugs-of-abuse and had clinically acceptable 12-lead electrocardiograms (ECG). Participants were excluded from the study due to tobacco use; certain viral infections (eg hepatitis and HIV); medication use; conditions that interfere with the absorption, distribution, metabolism, or excretion of drugs; and history of drug-induced rash.

Participants were randomly assigned to one of three cohorts and dosed to achieve steady state: (A) lamotrigine plus oxcarbazepine, (B) lamotrigine plus placebo, or (C) oxcarbazepine plus placebo. Lamotrigine $25-200 \mathrm{mg}$ or placebo was orally administered once daily according to the recommended 42-day up-titration regimen (ie $25 \mathrm{mg}$ on Days 1-14, $50 \mathrm{mg}$ on Days 15-28, $100 \mathrm{mg}$ on Days 29-35, $200 \mathrm{mg}$ on Days 36-42). On Day 43, all participants were randomly assigned to receive concomitant drugs per protocol on an in-patient basis. Oxcarbazepine was orally administered, twice daily at total doses of $600 \mathrm{mg}$ on Days 43 and 44, $900 \mathrm{mg}$ on Days 45 and 46, and $1200 \mathrm{mg}$ on Days 47-53.

Serial blood samples for determination of serum lamotrigine, plasma oxcarbazepine, and MHD concentrations were collected predose on Days 51-53 to confirm steadystate concentrations of lamotrigine, on Day 53 at regular intervals up to $24 \mathrm{~h}$ for lamotrigine, and up to $12 \mathrm{~h}$ for oxcarbazepine and MHD. Serum samples were assayed for lamotrigine by liquid chromatography mass spectroscopy (LC-MS-MS); the lower limit of quantification (LLQ) was $4 \mathrm{ng} / \mathrm{ml}$. Plasma samples were assayed for oxcarbazepine and MHD by HPLC/UV analysis. The LLQ for each were $0.1 \mu \mathrm{g} / \mathrm{ml}$. Pharmacokinetic parameters were determined by noncompartmental methods.

Compliance was ensured during the outpatient lamotrigine or placebo monotherapy period by pill counts during weekly clinic visits and by calling participants between clinic visits. During the in-patient monotherapy phase, drugs were administered by research personnel. Safety and tolerability was monitored throughout the study through regular assessments of vital signs, ECGs, and clinical laboratory test results. The subjects were encouraged to report any potential adverse events and were actively interrogated about any signs of adverse events at regular time points.

Prior to initiation of the study, an appropriate sample size was calculated based on the following considerations: A difference of $30 \%$ in the mean of AUC and $C_{\max }$ in absence and presence of the other drug was considered to be clinically significant taking into account that differences of $20 \%$ (means within a confidence interval of $80-125 \%$ ) are generally considered as bioequivalent even for narrow therapeutic range drugs (Food and Drug Administration, 2003). Values of between-subject coefficient of variation (CV) were based on the results from a previous lamotrigine repeat dose study in which estimates of the between-subject variability for log-transformed AUC and $C_{\max }$ of lamotrigine were approximately $23 \%$ for both AUC and $C_{\max }$ (unpublished results) - this was confirmed by the results of this study (Table 1). The CV estimates for AUC and $C_{\max }$ of oxcarbazepine and MHD were based on published studies (Keranen et al, 1992a,b). In these studies between-subject variability of the $\mathrm{PK}$ of the 10-monohydroxy metabolite (MHD) of oxcarbazepine was between 18 and 23\% for AUC and between 13 and $20 \%$ for $C_{\text {max }}$. Assuming a betweensubject CV of $23 \%$, it was estimated that a sample size of 10 subjects per arm will provide at least $90 \%$ power to detect a clinically significant difference of $30 \%$ in the PK of the MHD of oxcarbazepine and in the PK of lamotrigine.

Statistical analysis was performed by analysis of variance (ANOVA) fitting a term for regimen. $\log _{\mathrm{e}}$-transformed $\mathrm{AUC}_{(0-24)}$ and $C_{\max }$ of lamotrigine, in the presence and absence of oxcarbazepine, and $\log _{\mathrm{e}}$-transformed $\mathrm{AUC}_{(0-12)}$ and $C_{\max }$ of the MHD metabolite, in the presence and absence of lamotrigine, were separately analyzed. Data from regimens A (lamotrigine + oxcarbazepine) and B (lamotrigine alone) were used in the model for the effect of oxcarbazepine on lamotrigine and from regimens $\mathrm{A}$ and $\mathrm{C}$ (oxcarbazepine alone) for the model of the effect of lamotrigine on MHD.

To investigate the effects of lamotrigine on MHD, point estimates and $90 \%$ confidence intervals were constructed for the differences in $\mathrm{AUC}_{(0-12)}$ and $C_{\max }$ of $\mathrm{MHD}$ in the presence and absence of lamotrigine $(A-C)$, using the residual variance from the ANOVA. These were backtransformed to obtain the estimates for the ratio oxcarbazepine + lamotrigine: oxcarbazepine $(\mathrm{A}: \mathrm{C})$. The effects of oxcarbazepine on lamotrigine were similarly analyzed to obtain the estimates for the ratio oxcarbazepine + lamotrigine:lamotrigine $(\mathrm{A}: \mathrm{B})$. The results were expressed as the ratio of the pharmacokinetic parameters and the $90 \%$ confidence interval of this ratio.

To evaluate whether steady state was achieved for each treatment, separate statistical analyses of predose concentrations were performed for Days 51-54 after a $\log _{\mathrm{e}^{-}}$ transformation of the data. A mixed effect model was fitted with day as a fixed effect, subject as a random effect, and day as a continuous covariate. 
Using the pooled estimate of variance, the $90 \%$ confidence intervals (CI) for the slope of the day effect on the log scale were calculated and then back transformed. If both the back-transformed slope estimate and the $90 \%$ CI limits were within the range of $0.91-1.10$, then steady state was regarded as being statistically confirmed.

\section{RESULTS}

A total of 47 male, outpatient volunteers aged 19-54 years were randomly assigned to receive lamotrigine $(n=31)$ or placebo $(n=16)$. Of these, about half were randomly assigned to receive concomitant oxcarbazepine $(n=15)$ or placebo $(n=16)$. Subjects were predominantly Caucasian (97\%), with mean body weight ranging from 80.2 to $83.2 \mathrm{~kg}$ across treatment regimens, and mean BMI ranging from 25.1 to $26.1 \mathrm{~kg} / \mathrm{m}^{2}$ across treatment regimens. In all, 39 subjects completed the study providing a full set of pharmacokinetic data.

\section{Pharmacokinetics}

Pharmacokinetic profiles were obtained from 13 subjects in each cohort. Steady state was achieved in all groups by Day 51 as confirmed by the statistical analysis of predose concentration slopes (data not shown).
Oxcarbazepine had minimal effect on lamotrigine $\mathrm{AUC}_{(0-24)}$ and $C_{\max }$ at steady state (Figure 1, Table 1). Similarly, lamotrigine had no significant effect on systemic MHD or oxcarbazepine exposure (Figure 2, Table 1). Betweensubject variability for both lamotrigine and MHD was similar for coadministration and placebo treatment.

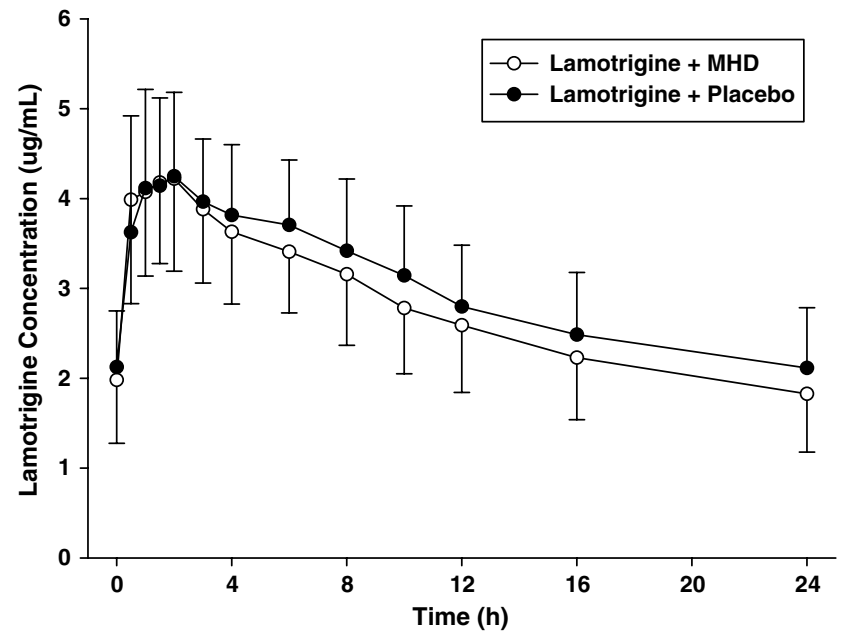

Figure I Mean steady-state serum lamotrigine concentration-time profiles. Error bars represent standard deviation.

Table I Pharmacokinetics of Lamotrigine, Oxcarbazepine, and MHD

\begin{tabular}{|c|c|c|c|c|}
\hline \multirow[b]{2}{*}{ LTG } & $\begin{array}{c}\text { Regimen A } \\
\quad+O X C\end{array}$ & $\begin{array}{c}\text { Regimen B } \\
\text { +PBO }\end{array}$ & \multirow{2}{*}{$\begin{array}{c}\text { Ratio }(90 \% \mathrm{Cl}) \\
\mathrm{A}: \mathrm{B}\end{array}$} & \multirow[b]{2}{*}{ CVb (\%) } \\
\hline & $(n=13)$ & $(n=13)$ & & \\
\hline$C_{\max }(\mathrm{ng} / \mathrm{ml})$ & $44 I I(2905-65 \mid 4)$ & 4479 (3176-5906) & $0.98(0.85,1.15)$ & 22.8 \\
\hline \multirow[t]{2}{*}{$t_{\max }(h)^{\mathrm{a}}$} & $1.00(0.50-2.00)$ & $1.50(0.50-4.00)$ & - & - \\
\hline & $\begin{array}{c}\text { Regimen A } \\
\text { +LTG }\end{array}$ & $\begin{array}{c}\text { Regimen C } \\
\text { +PBO }\end{array}$ & & \\
\hline MHD & $(n=13)$ & $(n=13)$ & $A: C$ & \\
\hline$t_{\max }(h)^{\mathrm{a}}$ & $\begin{array}{c}\text { Regimen A } \\
\text { +LTG }\end{array}$ & $\begin{array}{c}\text { Regimen C } \\
\text { +PBO }\end{array}$ & - & - \\
\hline Oxc & $(n=13)$ & $(n=13)$ & & \\
\hline$C_{\max }(\mu g / m l)$ & $1.52(0.73-2.95)$ & $1.66(0.91-2.74)$ & - & - \\
\hline 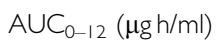 & $6.44(4.49-9.43)$ & 7.06 (5.00-9.03) & - & - \\
\hline$t_{\max }(h)^{\mathrm{a}}$ & $1.00(0.50-3.00)$ & $1.00(0.50-3.00)$ & - & - \\
\hline
\end{tabular}

aMedian (range).

$\mathrm{LTG}=$ lamotrigine; $\mathrm{MHD}=10$-monohydroxy metabolite of $\mathrm{OXC} ; \mathrm{OXC}=$ oxcarbazepine; $\mathrm{Cl}=$ confidence interval; $\mathrm{CVb}=$ between-subject variability. 

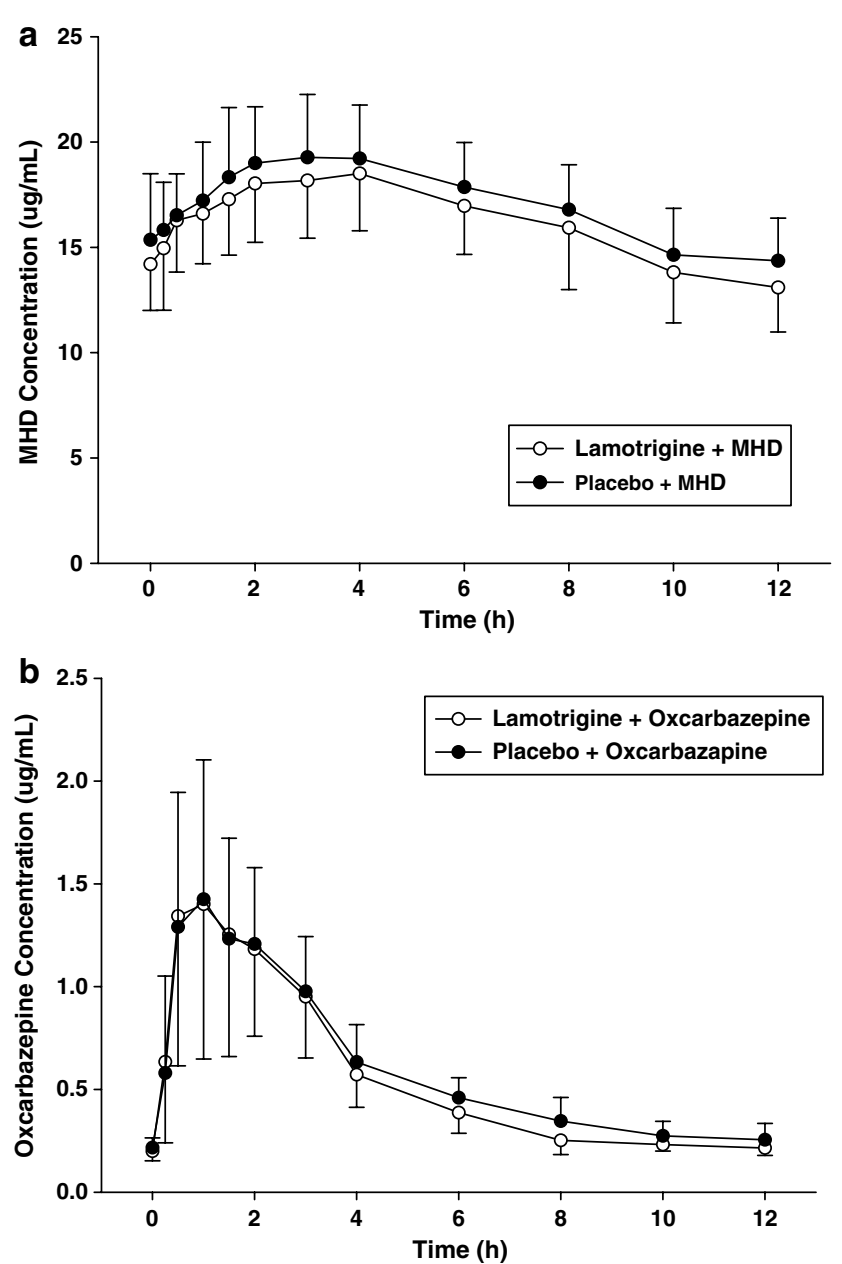

Figure 2 Mean steady-state plasma (a) MHD and (b) oxcarbazepine concentration-time profiles. Error bars represent standard deviation.

\section{Tolerability}

During the up-titration phase (Days 1-42), adverse events were comparable in the lamotrigine and placebo cohorts (Table 2). All adverse events were of mild intensity, except for two that were of moderate intensity (dental infection and pharyngitis). Two subjects withdrew during this phase: one subject receiving placebo withdrew due to rash and flu-like symptoms and one subject receiving lamotrigine withdrew due to rash that was secondary to a viral infection. All volunteers with rash, regardless of type or cause, were subject to protocol-specified study withdrawal.

During the polytherapy phase (Days 43-53), 44 adverse events occurred in the 15 subjects receiving lamotrigine and placebo; all adverse events were of mild intensity, except for one episode each of moderate intensity dry skin and headache. Two subjects were withdrawn, one because of a rash and one elected to withdraw because of mild nausea and vomiting.

The 14 subjects receiving oxcarbazepine and placebo reported 51 adverse events, all of mild intensity, except for a moderate intensity maculo-papular rash, which led to study withdrawal.

Adverse events occurred with greater frequency in the lamotrigine plus oxcarbazepine cohort (Table 2). Somnolence was exclusively reported in this cohort, and headache, nausea, and dizziness occurred with greater frequency in this cohort compared with the monotherapy cohorts of either drug. Five subjects reported a total of 11 adverse events of at least moderate intensity (two were judged severe) during combination therapy. Two subjects were withdrawn from this cohort due to adverse events that were probably related to treatment (a combination of dizziness, headache, abnormal vision, and purpura in one subject and vomiting in one subject).

Table 2 Most Common ( $\geqslant 25 \%$ of Subjects) Treatment Emergent Adverse Events

Monotherapy phase days I-42

\begin{tabular}{|c|c|c|c|c|c|c|}
\hline & & & & & & \\
\hline & Cohort I & Cohort 2 & Cohort 3 & $\begin{array}{c}\text { Cohort I } \\
\text { (Regimen A) }\end{array}$ & $\begin{array}{c}\text { Cohort } 2 \\
\text { (Regimen B) }\end{array}$ & $\begin{array}{c}\text { Cohort } 3 \\
\text { (Regimen C) }\end{array}$ \\
\hline & LTG & LTG & PBO & LTG+OXC & LTG+PBO & OXC+PBO \\
\hline & $n=15$ & $n=16$ & $n=16$ & $n=15$ & $n=15$ & $n=14$ \\
\hline Adverse event & $n^{a}(\%)^{b}$ & $n^{a}(\%)^{b}$ & $n^{a}(\%)^{b}$ & $n^{a}(\%)^{b}$ & $n^{a}(\%)^{b}$ & $n^{a}(\%)^{b}$ \\
\hline Nausea & $2(13)$ & $4(13)$ & 0 & $16(47)$ & $4(20)$ & $3(21)$ \\
\hline Dizziness & I (7) & I (6) & 0 & $17(60)$ & $3(13)$ & $7(43)$ \\
\hline Pharyngitis & 0 & $4(25)$ & $2(13)$ & $4(20)$ & $3(20)$ & $3(2 \mid)$ \\
\hline Rhinitis & $3(20)$ & $2(13)$ & $6(3 \mid)$ & $2(14)$ & $3(20)$ & I (7) \\
\hline
\end{tabular}

$\mathrm{AE}=$ adverse event; $\mathrm{LTG}=$ lamotrigine; $\mathrm{OXC}=$ oxcarbazepine; $\mathrm{PBO}=$ placebo

${ }^{\mathrm{a}}$ Total number of adverse events,

b\% of volunteers experiencing at least one adverse event.
Combination therapy phase days $43-53$ 
No clinically significant changes in clinical laboratory parameters, vital signs, or ECGs were reported during the study.

\section{DISCUSSION}

The results of this drug-interaction study of lamotrigine and oxcarbazepine in healthy subjects showed no pharmacokinetic interactions leading to higher drug concentrations. Coadministration of lamotrigine and oxcarbazepine, at approved doses, resulted in less than $10 \%$ reduction of systemic lamotrigine and MHD exposure. The lamotrigine findings are consistent with in vitro evidence that oxcarbazepine/MHD minimally induces the UDP-glucuronyltransferase system and that oxcarbazepine is a less potent hepatic enzyme-inducer than carbamazepine (Flesch, 2004).

The doses used in this study reflect approved dose levels (Lamictal ${ }^{\circledR}, 2004$; Trileptal $^{\circledR}$, 2004) or those used in previous drug-interaction studies (Fattore et al, 1999; Hulsman et al, 1995); however, they do not necessarily reflect actual clinical practice, particularly in epilepsy, where higher doses are often prescribed. While the current findings are restricted to the doses studied, it is unlikely that the metabolism and excretion of higher doses will greatly differ.

To allow sufficient time to observe enzyme induction at a clinically relevant scale, a 10-day cotherapy period based on the terminal half-life of lamotrigine (approximately $25 \mathrm{~h}$ after repeated doses) (Lamictal $\left.{ }^{\circledR}, 2004\right)$, oxcarbazepine and MHD (approximately 2 and $9 \mathrm{~h}$, respectively) (Lloyd et al, 1994) and an estimation of the necessary induction time was used. Of these 10 days, four were oxcarbazepine dose escalation days resulting in 6 days of cotherapy at maximum doses. Time to induction and reversal of induction upon removal of the inducer are dependent on the rate of enzyme synthesis and degradation and time to steady-state plasma concentration of the inducer (ie five elimination half-lives) (Park and Breckenridge, 1981). Data investigating the turnover of UDP-glucuronyl transferase, the metabolizing enzyme of lamotrigine and MHD, are not available. However, it is known that cytochrome P450 3A function has a recovery half-life of $23 \mathrm{~h}$, with a largely complete recovery after 3 days (Greenblatt et al, 2003). In a study of metabolism-inducers, the rate-limiting step for enzyme induction was the half-life of the inducer (Ohnhaus et al, 1989), which is relatively short for oxcarbazepine or MHD. The statistical confirmation that each compound had achieved steady-state prior to obtaining the pharmacokinetic profile adds further evidence that a sufficient duration of cotherapy to demonstrate induction had taken place.

Importantly, these current prospectively determined findings did not detect the pharmacokinetic interactions reported in several retrospective studies. May et al (1999) reported a significant $30 \%$ reduction in lamotrigine plasma concentration with coadministration of oxcarbazepine, while Kramer et al (2003) reported a 34\% reduction. Further, Guenault et al (2003) reported that lamotrigine significantly reduced the serum concentration of oxcarbazepine by a factor of 2.21 . As discussed, our study, while carefully designed, is limited by the selected doses, the duration of cotherapy, and the inclusion of healthy subjects instead of patients. However, retrospective data are often less robust than data derived from prospective, controlled studies of healthy subjects, as they are derived from clinical records and may reflect poor patient compliance, resulting in lower drug concentrations, or may be confounded by concomitant medication use, diet, and varying dosage times relative to pharmacokinetic sampling. Additionally, data indicating a drug interaction are more likely to be published than data indicating no interaction (Callaham et al, 1998).

The adverse events observed in the present study during monotherapy are similar to those previously described for lamotrigine and oxcarbazepine $\left(\right.$ Lamictal ${ }^{\circledR}$, 2004; Trileptal $\left.{ }^{\circledR}, 2004\right)$. Adverse events were observed more frequently and with a slightly greater intensity when lamotrigine and oxcarbazepine were coadministered. Two subjects were withdrawn from this treatment regimen for potentially drug-related adverse events. When discounting the protocol-specified withdrawals due to rash, this number of withdrawals related to adverse events is higher than in the other treatment regimens. Interpretation of these data unfortunately is limited by the small sample size of this study.

As both are potent CNS-active drugs with well-described adverse event profiles, the combination could have resulted in additive pharmacodynamic effects and adverse events. Although a greater incidence of adverse events in the combination therapy cohort compared with the monotherapy cohorts was expected, tolerability of monotherapy and polytherapy cannot be directly compared because total drug loads were unequal (Deckers et al, 1997). Doses of each drug in the combination therapy cohort would have to be halved to equalize the drug loads in the monotherapy cohort. Therefore, the increased incidences and severity of adverse events with full doses of each drug may not have been caused by an interactive potentiation of pharmacodynamic drug effects. They more likely indicate additive drug effects due to a higher drug load in the combination therapy cohort.

In conclusion, this study supports the coadministration of lamotrigine and oxcarbazepine when clinically indicated. There is no inhibitory pharmacokinetic drug interaction. A mild induction of lamotrigine metabolism cannot be excluded but is unlikely to be of an extent requiring dose adjustments. Adverse events are more frequent in the cohort receiving the combination of lamotrigine and oxcarbazepine, which highlights the importance of monitoring the tolerability of the combination in clinical practice.

\section{REFERENCES}

Anderson GD, Gidal BE, Messenheimer JA, Gilliam FG (2002). Time course of lamotrigine de-induction: impact of step-wise withdrawal of carbamazepine or phenytoin. Epilepsy Res 49: 211-217.

Callaham ML, Wears RL, Weber EJ, Barton C, Young G (1998). Positive-outcome bias and other limitations in the outcome of research abstracts submitted to a scientific meeting. JAMA 280: 254-257 [Erratum in: JAMA (1998) 280: 1232.].

Cohen AF, Land GS, Breimer DD, Yuen WC, Winton C, Peck AW (1987). Lamotrigine, a new anticonvulsant: pharmacokinetics in normal humans. Clin Pharmacol Ther 42: 535-541. 
Deckers CL, Hekster YA, Keyser A, Meinardi H, Renier WO (1997). Drug load in clinical trials: a neglected factor. Clin Pharmacol Ther 62: 592-595.

Deckers CLP (2002). The place of combination therapy in the early treatment of epilepsy. CNS Drugs 16: 155-163.

Fattore C, Cipolla G, Gatti G, Limido GL, Sturm Y, Bernasconi C et al (1999). Induction of ethinylestradiol and levonorgestrel metabolism by oxcarbazepine in healthy women. Epilepsia 40: 783-787.

Flesch G (2004). Overview of the clinical pharmacokinetics of oxcarbazepine. Clin Drug Invest 24: 185-203.

Food and Drug Administration (2003). Guidance for Industry: Bioavailability and Bioequivalence Studies for Orally Administered Drug Products-General Considerations. http://www.fda. gov/cder/guidance/index.htm.

Glauser TA (2001). Oxcarbazepine in the treatment of epilepsy. Pharmacotherapy 21: 904-919.

Goa KL, Ross SR, Chrisp P (1993). Lamotrigine. A review of its pharmacological properties and clinical efficacy in epilepsy. Drugs 46: 152-176.

Greenblatt DJ, von Moltke LL, Harmatz JS, Chen G, Weemhoff JL, Jen C. et al (2003). Time course of recovery of cytochrome p450 $3 \mathrm{~A}$ function after single doses of grapefruit juice. Clin Pharmacol Ther 74: 121-129.

Guenault N, Odou P, Robert H (2003). Increase in dihydroxycarbamazepine serum levels in patients co-medicated with oxcarbazepine and lamotrigine. Eur J Clin Pharmacol 59: 781-782.

Hirschfeld RM, Kasper S (2004). A review of the evidence for carbamazepine and oxcarbazepine in the treatment of bipolar disorder. Int J Neuropsychopharmacol 30: 1-16 [Epub ahead of print].

Hulsman JARJ, Rentmeester TW, Banfield CR, Reidenberg P, Colucci RD, Meehan JW et al (1995). Effects of felbamate on the pharmacokinetics of the monohydroxy and dihydroxy metabolites of oxcarbazepine. Clin Pharmacol Ther 58: 383-389.

Keranen T, Jolkkonen J, Jensen PK, Menge GP, Andersson P (1992a). Absence of interaction between oxcarbazepine and erythromycin. Acta Neurol Scand 86: 120-123.

Keranen T, Jolkkonen J, Klosterskov-Jensen P, Menge GP (1992b). Oxcarbazepine does not interact with cimetidine in healthy volunteers. Acta Neurol Scand 85: 239-242.

Kramer G, Dorn T, Etter H (2003). Oxcarbazepine: clinically relevant drug interaction with lamotrigine. Epilepsia 44(Suppl 9): 95-96; Abstract No. 1.267. Presented at the Annual Meeting of the American Epilepsy Society. Boston, MA, USA, 05 December 2003-10 December 2003.

Lamictal $^{\mathbb{R}}$ (lamotrigine) [package insert] (2004) GlaxoSmithKline: Research Triangle Park, NC.

Lloyd P, Flesch G, Dieterle W (1994). Clinical pharmacology and pharmacokinetics of oxcarbazepine. Epilepsia 35(Suppl 3): S10-S13.

May TW, Korn-Merker E, Rambeck B (2003). Clinical pharmacokinetics of oxcarbazepine. Clin Pharmacokinet 42: 1023-1042.

May TW, Rambeck B, Jurgens U (1999). Influence of oxcarbazepine and methsuximide on lamotrigine concentrations in epileptic patients with and without valproic acid comedication: results of a retrospective study. Ther Drug Monit 21: 175-181.

Ohnhaus EE, Breckenridge AM, Park BK (1989). Urinary excretion of 6 beta-hydroxycortisol and the time course measurement of enzyme induction in man. Eur J Clin Pharmacol 36: 39-46.

Park BK, Breckenridge AM (1981). Clinical implications of enzyme induction and enzyme inhibition. Clin Pharmacokinet 6: 1-24.

Shorvon S (2000). Oxcarbazepine: a review. Seizure 9: 75-79.

Tecoma ES (1999). Oxcarbazepine. Epilepsia 40(Suppl 5): S37-S46.

Trileptal $^{\mathbb{R}}$ (oxcarbazepine) [package insert] (2004) Novartis: East Hanover, NJ. 